very interesting results as a consequence of the investigation of areas of audibility and inaudibility surrounding great sources of sound, such as the blasting for the Jungfraubahn, the bombardment of Antwerp, a munition explosion in England, etc. It seems natural that the Halifax explosion, violent enough to break glass many miles distant and to be heard scores of miles away at sea, should be investigated the same way; but I have read and heard nothing of any such study. It is, of course, a matter for the scientists of the neighboring region, and perhaps they have taken it up.

WiLlard J. FISHer

\section{PRIMITIVE KNOWLEDGE OF INOCULATION}

$\mathrm{I}_{\mathrm{N}}$ an article on "The Origin of the Custom of Tea Drinking in China," ScIence, March 15, R. A. Gortner remarks that "it is extremely improbable that it was recognized centuries ago that typhoid fevers, etc., were disseminated by pollution of the water supply, especially inasmuch as there was no knowledge of microorganisms or of the rôle which they play in disease until the work of Pasteur (1857-1863)." In adopting this conclusion as a priori valid it seems to me that Gortner is in danger of making the same error that was made by Sir Richard Burton in 1854. Burton states ("First Footsteps in East Africa") that "The mosquito bites bring on, according to the same authority (the Somal), deadly fevers; the superstition probably arises from the fact that mosquitoes and fevers become formidable about the same time." This is not the only case, we may be sure, in which causal relations have been recognized long before the causal mechanism was known.

Knight Dunlap

\section{SCIENTIFIC BOOKS}

The Anthocyanin Pigments of Plants. By Muriel Wheldale. Cambridge University Press. 1916. Royal 8vo. Pp. xii +318 . Price 15 s net.

The science of chemistry has grown so rapidly during recent years that it is im- possible for an individual to acquire a thorough knowledge of all of its branches, and even to master a single phase of the science often means laborious searchings through the chemical literature. Fortunately there have appeared during the last decade a number of monographs, each written by an authority in that particular field, which deal thoroughly with a special topic and sum up all of the available literature. Such a compilation is the present volume.

What causes the production of the colors in a flower? Every one has asked himself the question and numerous chemists have attacked the problem, yet it is only within recent years that any definite knowledge has been attained and we still have a long way to progress before we know the whole truth. It is fortunate, however, that Miss Wheldale has accumulated such evidence as is at present available.

Her studies of anthocyanin began with a study of the genetical behavior of these pigments, but she soon ascertained that biological phenomena have for their basis chemical reactions, with the result that she undertook to analyze the chemical changes which were involved in the hereditary behavior of flower coloration. The present volume is divided into two parts. Under Part I., "General Account of Anthocyanins," we have "Introductory," consisting mainly of the older literature of the subject; "The Morphological Distribution of Anthocyanins"; "The Histological Distribution of Anthocyanins"; "The Properties and Reactions of Anthocyanins" ; "The Isolation and Constitution of Anthocyanins"; "Physiological Conditions and Factors Influencing the Formation of Anthocyanins"; "Reactions Involved in the Formation of Anthocyanins"; and "The Significance of Anthocyanins," practically all of which are taken up from the chemical viewpoint.

Under Part II., "Anthocyanin and Genetics," we find "Classes of Variation"; "Details of Cases of Mendelian Inheritance in Color Varieties"; "Connection of Flower Color with the Presence of Anthocyanin Vegetative Organs, Fruits and Seeds"; "Heterozygous Forms"; "Color Factors in Reduplication 